\title{
Two-Dimensional Measure Vector of Departure from Marginal Homogeneity in Square Contingency Tables
}

\author{
Shuji Ando ${ }^{1^{*}}$, Takashi Takeuchi ${ }^{2},{\text { Kiyotaka } \mathrm{Iki}^{3} \text { and Sadao Tomizawa }}^{2}$ \\ ${ }^{1}$ Department of Information and Computer Technology, Faculty of Engineering, \\ Tokyo University of Science, Katsushika-ku, Tokyo, Japan \\ ${ }^{2}$ Department of Information Sciences, Faculty of Science and Technology, \\ Tokyo University of Science, Noda City, Chiba, Japan \\ ${ }^{3}$ Department of Economics, Nihon University, Chiyoda-ku, Tokyo, Japan \\ Email: shuji.ando@rs.tus.ac.jp
}

\begin{abstract}
For square contingency tables with ordered categories, Tahata et al. (2006) and Tahata et al. (2012) considered measures which represent the degree of departure from the marginal homogeneity $(\mathrm{MH})$ model using the cumulative marginal probabilities. The former measure can judge whether or not the MH model holds, but the latter cannot. Also, the latter measure can distinguish the directionality for two types of maximum marginal inhomogeneities, but the former cannot. The present paper proposes a two-dimensional measure vector with above measures as elements that can simultaneously analyze the degree of departure from $\mathrm{MH}$ and the directionality for two types of maximum marginal inhomogeneities. Also, this paper shows the usefulness of the proposed measure vector on comparing visually degrees of departure from $\mathrm{MH}$ in several square contingency tables using confidence region.
\end{abstract}

Keywords: Comparison, confidence region, ordinal category, visualized measure.

\section{Introduction}

Consider an $r \times r$ square contingency table with ordered categories. Let $p_{i j}$ denote the probability that an observation will fall in the $i$ th row and $j$ th column of the table $(i=1, \ldots, r ; j=1, \ldots, r)$, and let $X$ and $Y$ denote the row and column variables, respectively.

The symmetry (S) model (Bowker, 1948) is defined by

$$
\operatorname{Pr}(X=i, Y=j)=\operatorname{Pr}(X=j, Y=i) \quad(i \neq j),
$$

namely

$$
p_{i j}=p_{j i} \quad(i \neq j) .
$$

For representing the degree of departure from the S model, Tomizawa, Miyamoto and Hatanaka (2001) proposed a measure $\gamma$ (although the detail is omitted), and Tahata, Miyazawa and Tomizawa (2010) proposed a measure $\phi$ (although the detail is omitted). Also, Ando, Tahata and Tomizawa (2017) proposed a two-dimensional measure vector $\boldsymbol{\Phi}=(\gamma, \phi)^{t}$ to represent the degree of departure from the $\mathrm{S}$ model. Note that the symbol " $t$ " denotes the transpose.

The marginal homogeneity (MH) model (Stuart, 1955) is defined by

$$
\operatorname{Pr}(X=i)=\operatorname{Pr}(Y=i) \quad(i=1, \ldots, r),
$$

namely

$$
p_{i \cdot}=p_{\cdot i} \quad(i=1, \ldots, r),
$$

where

$$
p_{i .}=\sum_{t=1}^{r} p_{i t}, \quad p_{\cdot i}=\sum_{s=1}^{r} p_{s i}
$$

This model states that the row marginal distribution is equal to the column marginal distribution. 
Let $F_{i}^{X}$ and $F_{i}^{Y}$ denote the cumulative marginal probabilities of $X$ and $Y$, respectively; those are

$$
F_{i}^{X}=\operatorname{Pr}(X \leq i)=\sum_{k=1}^{i} p_{k}, \quad F_{i}^{Y}=\operatorname{Pr}(Y \leq i)=\sum_{k=1}^{i} p_{\cdot k} \quad(i=1, \ldots, r-1)
$$

Using the cumulative marginal probabilities $\left\{F_{i}^{X}, F_{i}^{Y}\right\}$, the $\mathrm{MH}$ model can be expressed as

$$
F_{i}^{X}=F_{i}^{Y} \quad(i=1, \ldots, r-1)
$$

This states that the row cumulative marginal distribution is identical with the column cumulative marginal distribution.

When the MH model does not hold, we are interested in measuring the degree of departure from $\mathrm{MH}$. For square contingency tables with ordered categories, Tahata, Iwashita and Tomizawa (2006) proposed a measure based on the cumulative marginal probabilities (denoted by $\Omega$ in Section 2) which represents the degree of departure from MH. The measure $\Omega$ has characteristics that (i) $\Omega$ lies between 0 and 1 , (ii) $\Omega=0$ if and only if the MH model holds, and (iii) $\Omega=1$ if and only if the degree of departure from MH is the maximum (say, maximum marginal inhomogeneity), in the sense that $F_{i}^{X}=0\left(\right.$ then $F_{i}^{Y} \neq 0$ ) for all $i=1, \ldots, r-1$ (say, maximum lower-marginal inhomogeneity) or $F_{i}^{Y}=0$ (then $F_{i}^{X} \neq 0$ ) for all $i=1, \ldots, r-1$ (say, maximum right-marginal inhomogeneity). For example, the measure $\Omega$ takes the maximum value 1 in the sense of maximum lower-marginal inhomogeneity and maximum right-marginal inhomogeneity for the cases in Tables $1 \mathrm{a}$ and $1 \mathrm{~b}$, respectively. The measure $\Omega$ can judge whether or not the MH model holds. However, the measure $\Omega$ cannot distinguish the directionality for two types of maximum marginal inhomogeneities (i.e., maximum lower-marginal inhomogeneity or maximum rightmarginal inhomogeneity) because of property (iii).

\begin{tabular}{|c|c|c|c|c|c|c|c|c|c|c|c|}
\hline & (1) & $(2)$ & $(3)$ & $(4)$ & Total & & (1) & $(2)$ & $(3)$ & $(4)$ & Total \\
\hline \multicolumn{6}{|c|}{ (a) Lower-marginal inhomogeneity } & \multicolumn{6}{|c|}{ (c) Upper-marginal inhomogeneity } \\
\hline (1) & 0 & 0 & 0 & 0 & 0 & (1) & 0.4 & 0.3 & 0.2 & 0.1 & 1 \\
\hline (2) & 0 & 0 & 0 & 0 & 0 & $(2)$ & 0 & 0 & 0 & 0 & 0 \\
\hline (3) & 0 & 0 & 0 & 0 & 0 & (3) & 0 & 0 & 0 & 0 & 0 \\
\hline (4) & 0.4 & 0.3 & 0.2 & 0.1 & 1 & (4) & 0 & 0 & 0 & 0 & 0 \\
\hline Total & 0.4 & 0.3 & 0.2 & 0.1 & 1 & Total & 0.4 & 0.3 & 0.2 & 0.1 & 1 \\
\hline \multicolumn{6}{|c|}{ (b) Right-marginal inhomogeneity } & \multicolumn{6}{|c|}{ (d) Left-marginal inhomogeneity } \\
\hline (1) & 0 & 0 & 0 & 0.4 & 0.4 & (1) & 0.4 & 0 & 0 & 0 & 0.4 \\
\hline$(2)$ & 0 & 0 & 0 & 0.3 & 0.3 & (2) & 0.3 & 0 & 0 & 0 & 0.3 \\
\hline (3) & 0 & 0 & 0 & 0.2 & 0.2 & (3) & 0.2 & 0 & 0 & 0 & 0.2 \\
\hline (4) & 0 & 0 & 0 & 0.1 & 0.1 & (4) & 0.1 & 0 & 0 & 0 & 0.1 \\
\hline Total & 0 & 0 & 0 & 1 & 1 & Total & 1 & 0 & 0 & 0 & 1 \\
\hline
\end{tabular}

Table 1. The examples of artificial probabilities with the maximum departure from $\mathrm{MH}$.

Tahata, Kawasaki and Tomizawa (2012) proposed a measure (denoted by $\Psi$ in Section 2 ) which can distinguish the directionality for two types of maximum marginal inhomogeneities. The measure $\Psi$ has characteristics that (i) $\Psi$ lies between -1 and 1 , (ii) $\Psi=-1$ if and only if there is a structure of maximum right-marginal inhomogeneity, (iii) $\Psi=1$ if and only if there is a structure of maximum lower-marginal inhomogeneity, and (iv) if the MH model holds then $\Psi=0$, but the converse does not hold. The measure $\Psi$ cannot directly measure the degree of departure from $\mathrm{MH}$ because of property (iv) (although the measure $\Psi$ can distinguish the directionality for two types of maximum marginal inhomogeneities).

In a similar manner to the measure vector $\boldsymbol{\Phi}=(\gamma, \phi)^{t}$ to represent the degree of departure from the $\mathrm{S}$ model, we are interested in the measure vector which can judge whether or not the MH model holds and distinguish the directionality for two types of maximum marginal inhomogeneities simultaneously. 
The purpose of this paper is to propose the measure vector which represents the degree of departure from $\mathrm{MH}$ including directionality for two types of maximum marginal inhomogeneities. The proposed measure vector would be useful for comparing visually degrees of departure from $\mathrm{MH}$ in several tables.

\section{Measure Vector}

Let

$$
F_{1(i)}^{*}=\frac{F_{i}^{X}}{\Delta}, F_{2(i)}^{*}=\frac{F_{i}^{Y}}{\Delta}, Q_{i}^{*}=\frac{1}{2}\left(F_{1(i)}^{*}+F_{2(i)}^{*}\right) \quad(i=1, \ldots, r-1)
$$

and

$$
\Delta=\sum_{i=1}^{r-1}\left(F_{i}^{X}+F_{i}^{Y}\right)
$$

Assume that $\left\{F_{i}^{X}+F_{i}^{Y} \neq 0\right\}$, we propose a measure vector which represents the degree of departure from $\mathrm{MH}$ defined by

$$
\boldsymbol{\Lambda}=\left(\begin{array}{c}
\Omega \\
\Psi
\end{array}\right) ; \text { the } 2 \times 1 \text { vector }
$$

where

$$
\begin{gathered}
\Omega=\frac{1}{\log 2} I_{M H} \\
\Psi=\frac{4}{\pi} \sum_{i=1}^{r-1}\left(F_{1(i)}^{*}+F_{2(i)}^{*}\right)\left(\theta_{i}-\frac{\pi}{4}\right),
\end{gathered}
$$

with

$$
\begin{gathered}
I_{M H}=\sum_{i=1}^{r-1}\left[F_{1(i)}^{*} \log \left(\frac{F_{1(i)}^{*}}{Q_{i}^{*}}\right)+F_{2(i)}^{*} \log \left(\frac{F_{2(i)}^{*}}{Q_{i}^{*}}\right)\right], \\
\theta_{i}=\sin ^{-1}\left(\frac{F_{i}^{Y}}{\sqrt{\left(F_{i}^{X}\right)^{2}+\left(F_{i}^{Y}\right)^{2}}}\right) .
\end{gathered}
$$

Note that $I_{M H}$ is the Kullback-Leibler information between $\left\{F_{1(i)}^{*}, F_{2(i)}^{*}\right\}$ and $\left\{Q_{i}^{*}, Q_{i}^{*}\right\}$. Measures $\Omega$ and $\Psi$ are given by Tahata et al. (2006) and Tahata et al. (2012), respectively.

From characteristics of measures $\Omega$ and $\Psi$, we obtain the following theorem.

\section{Theorem 1.}

(i) $\boldsymbol{\Lambda}=(0,0)^{t}$ if and only if the MH model holds,

(ii) $\boldsymbol{\Lambda}=(1,-1)^{t}$ if and only if there is a structure of maximum right-marginal inhomogeneity,

(iii) $\boldsymbol{\Lambda}=(1,1)^{t}$ if and only if there is a structure of maximum lower-marginal inhomogeneity.

Therefore, we see that the measure vector $\boldsymbol{\Lambda}$ can measure the degree of departure from $\mathrm{MH}$ which includes directionality for two types of maximum marginal inhomogeneities.

\section{Approximate Confidence Region for Measure Vector}

Let $n_{i j}$ denote the observed frequency in the $i$ th row and $j$ th column of the table $(i=1, \ldots, r ; j=$ $1, \ldots, r)$. Assume that a multinomial distribution applies to the $r \times r$ table. The sample version of $\Omega$, i.e., $\widehat{\Omega}$, is given by $\Omega$ with $\left\{p_{i j}\right\}$ replaced by $\left\{\hat{p}_{i j}\right\}$, where $\hat{p}_{i j}=n_{i j} / n$ and $n=\sum \sum n_{i j}$. Similarly, the sample version of $\Psi$, i.e., $\widehat{\Psi}$, is given by $\Psi$ with $\left\{p_{i j}\right\}$ replaced by $\left\{\hat{p}_{i j}\right\}$. Therefore, the sample version of $\boldsymbol{\Lambda}$, i.e., $\widehat{\Lambda}$, is given by $\boldsymbol{\Lambda}$ with $\Omega$ and $\Psi$ replaced by $\widehat{\Omega}$ and $\widehat{\Psi}$, respectively. Using the delta method (see, e.g., Agresti, 2013, p.590), we obtain the following theorem. 


\section{Theorem 2.}

$\sqrt{n}(\widehat{\boldsymbol{\Lambda}}-\boldsymbol{\Lambda})$ has asymptotically (as $n \rightarrow \infty$ ) a bivariate normal distribution with mean zero vector and the covariance matrix $\boldsymbol{\Sigma}[\widehat{\boldsymbol{\Lambda}}]$, where

$$
\boldsymbol{\Sigma}[\widehat{\boldsymbol{\Lambda}}]=\sum_{s=1}^{r} \sum_{t=1}^{r}\left(\begin{array}{c}
p_{s t}\left(w_{s t}\right)^{2} p_{s t} w_{s t} a_{s t} \\
p_{s t} w_{s t} a_{s t} p_{s t}\left(a_{s t}\right)^{2}
\end{array}\right),
$$

with

$$
\begin{aligned}
w_{s t} & =\frac{1}{\Delta \log 2}\left[\sum_{i=1}^{r-1}\left\{I(s \leq i) \log \left(\frac{F_{i}^{X}}{F_{i}^{X}+F_{i}^{Y}}\right)+I(t \leq i) \log \left(\frac{F_{i}^{Y}}{F_{i}^{X}+F_{i}^{Y}}\right)\right\}\right. \\
& -(2 r-(s+t))(\log 2)(\Omega-1)], \\
a_{s t}= & \frac{4}{\pi \Delta} \sum_{i=1}^{r-1}\left[\{I(s \leq i)+I(t \leq i)\} \theta_{i}+\frac{F_{i}^{X}+F_{i}^{Y}}{\left(F_{i}^{X}\right)^{2}+\left(F_{i}^{Y}\right)^{2}}\left\{-I(s \leq i) F_{i}^{Y}+I(t \leq i) F_{i}^{X}\right\}\right] \\
- & \frac{\{2 r-(s+t)\}(\Psi+1)}{\Delta},
\end{aligned}
$$

and $I(\cdot)$ is the indicator function, $I(\cdot)=1$ if true, 0 if not.

Let $\widehat{\boldsymbol{\Sigma}[\boldsymbol{\Lambda}}]$ denote $\boldsymbol{\Sigma}[\widehat{\boldsymbol{\Lambda}}]$ with $\left\{p_{i j}\right\}$ replaced by $\left\{\hat{p}_{i j}\right\}$. From Theorem 2, an approximate $100(1-p)$ percent confidence region for $\boldsymbol{\Lambda}$ is expressed as

$$
n(\widehat{\boldsymbol{\Lambda}}-\boldsymbol{\Lambda})^{t}(\widehat{\boldsymbol{\Sigma}[\boldsymbol{\Lambda}]})^{-1}(\widehat{\boldsymbol{\Lambda}}-\boldsymbol{\Lambda}) \leq \chi_{(1-p ; 2)}^{2},
$$

where $\chi_{(1-p ; 2)}^{2}$ is the $1-p$ quantile of the chi-square distribution with two degrees of freedom.

Consider comparison between degrees of departure from $\mathrm{MH}$ in Tables A and B (with sample sizes $n_{A}$ and $n_{B}$, respectively). For Tables $\mathrm{A}$ and $\mathrm{B}$, the measure vectors $\boldsymbol{\Lambda}$ are denoted by $\boldsymbol{\Lambda}_{A}$ and $\boldsymbol{\Lambda}_{B}$, respectively and its sample version are denoted by $\widehat{\boldsymbol{\Lambda}}_{A}$ and $\widehat{\boldsymbol{\Lambda}}_{B}$, respectively. When $n_{A}$ and $n_{B}$ are large, $\widehat{\boldsymbol{\Lambda}}_{A}-\widehat{\boldsymbol{\Lambda}}_{B}-\left(\boldsymbol{\Lambda}_{A}-\boldsymbol{\Lambda}_{B}\right)$ has approximately a bivariate normal distribution with mean zero vector and covariance matrix $\boldsymbol{V}$, where

$$
\boldsymbol{V}=\frac{1}{n_{A}} \boldsymbol{\Sigma}\left[\widehat{\boldsymbol{\Lambda}}_{A}\right]+\frac{1}{n_{B}} \boldsymbol{\Sigma}\left[\widehat{\boldsymbol{\Lambda}}_{B}\right] .
$$

Therefore, an approximate 100(1-p) percent confidence region for $\boldsymbol{\Lambda}_{A}-\boldsymbol{\Lambda}_{B}$ is expressed as

$$
\left(\widehat{\boldsymbol{\Lambda}}_{A}-\widehat{\boldsymbol{\Lambda}}_{B}-\left(\boldsymbol{\Lambda}_{A}-\boldsymbol{\Lambda}_{B}\right)\right)^{t} \widehat{\boldsymbol{V}}^{-1}\left(\widehat{\boldsymbol{\Lambda}}_{A}-\widehat{\boldsymbol{\Lambda}}_{B}-\left(\boldsymbol{\Lambda}_{A}-\boldsymbol{\Lambda}_{B}\right)\right) \leq \chi_{(1-p ; 2)}^{2},
$$

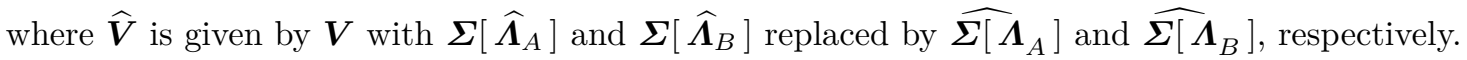

\section{Examples}

\subsection{Example 1}

Consider the data in Table 2 taken from Tominaga (1979, p.131). These data describe the crossclassification of father's and son's occupational status categories in Japan which were examined in 1955 and 1975. Note that status (1) is Professional and Managers, (2) is Clerical and Sales, (3) is Skilled manual and Semiskilled manual and (4) is Unskilled manual and Farmers. Thus, for the data in Tables $2 \mathrm{a}$ and $2 \mathrm{~b}$, the structure of lower-marginal inhomogeneity shows that son's status would be higher than father's, and the structure of right-marginal inhomogeneity shows that son's status would be lower than father's. 
Table 2. Occupational status for Japanese father-son pair's; from Tominaga (1979, p.131). Note that status (1) is Professional and Managers, (2) is Clerical and Sales, (3) is Skilled manual and Semiskilled manual and (4) is Unskilled manual and Farmers.

\begin{tabular}{|c|c|c|c|c|c|}
\hline \multirow[b]{2}{*}{ Father's status } & \multicolumn{4}{|c|}{ Son's status } & \multirow[b]{2}{*}{ Total } \\
\hline & $(1)$ & $(2)$ & $(3)$ & $(4)$ & \\
\hline \multicolumn{6}{|c|}{ (a) Examined in 1955} \\
\hline (1) & 80 & 72 & 37 & 19 & 208 \\
\hline (2) & 44 & 155 & 61 & 31 & 291 \\
\hline (3) & 26 & 73 & 218 & 45 & 362 \\
\hline (4) & 69 & 156 & 166 & 614 & 1005 \\
\hline Total & 219 & 456 & 482 & 709 & 1866 \\
\hline \multicolumn{6}{|c|}{ (b) Examined in 1975} \\
\hline (1) & 127 & 101 & 54 & 12 & 294 \\
\hline (2) & 86 & 207 & 125 & 13 & 431 \\
\hline (3) & 78 & 124 & 310 & 24 & 536 \\
\hline (4) & 109 & 206 & 437 & 325 & 1077 \\
\hline Total & 400 & 638 & 926 & 374 & 2338 \\
\hline
\end{tabular}

First, we shall measure degrees of departure from $\mathrm{MH}$ in Tables $2 \mathrm{a}$ and $2 \mathrm{~b}$ using the confidence region for $\boldsymbol{\Lambda}$. For Tables $2 \mathrm{a}$ and $2 \mathrm{~b}$, estimates of $\boldsymbol{\Lambda}$ are

$$
\widehat{\boldsymbol{\Lambda}}_{2 a}=\left(\begin{array}{c}
0.014 \\
0.169
\end{array}\right) \quad \text { and } \quad \widehat{\boldsymbol{\Lambda}}_{2 b}=\left(\begin{array}{c}
0.029 \\
0.248
\end{array}\right)
$$

respectively, and estimates of $\boldsymbol{\Sigma}$ are

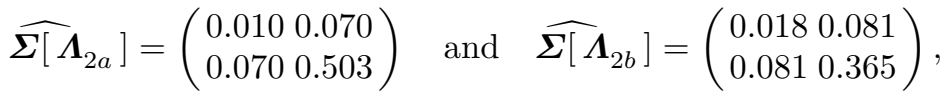

respectively.

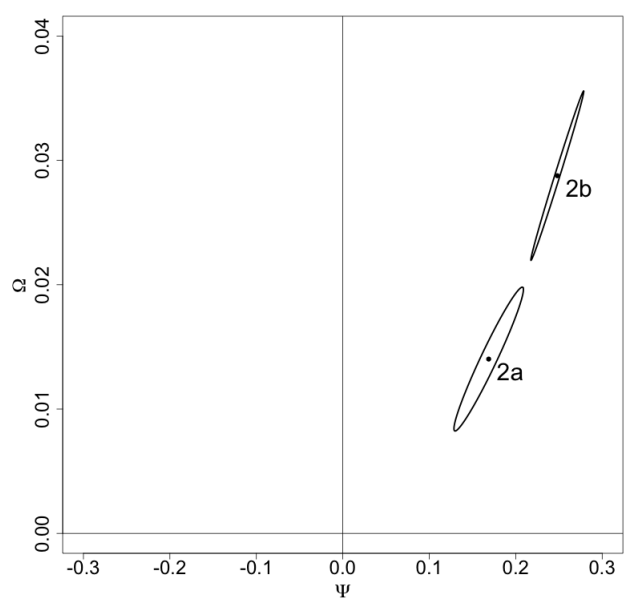

Figure 1. Approximate $95 \%$ confidence regions for $\boldsymbol{\Lambda}$, applied to Tables $2 \mathrm{a}$ and $2 \mathrm{~b}$. 
Confidence regions do not overlap for Tables $2 \mathrm{a}$ and $2 \mathrm{~b}$ (see Figure 1). From Figure 1, we can see that the data in both Tables $2 \mathrm{a}$ and $2 \mathrm{~b}$ tend to have a structure of lower-marginal inhomogeneity rather than the structure of right-marginal inhomogeneity. Thus, we see that son's status would be higher than father's in both 1955 and 1975.

Next, we shall compare between degrees of departure from $\mathrm{MH}$ in Tables $2 \mathrm{a}$ and $2 \mathrm{~b}$ using the confidence region for $\boldsymbol{\Lambda}_{2 a}-\boldsymbol{\Lambda}_{2 b}$. Note that, for these data, (i) if the confidence region for $\boldsymbol{\Lambda}_{2 a}-\boldsymbol{\Lambda}_{2 b}$ is included within the first quadrant in Figure 2, we can see that the degree of departure from MH in Table $2 \mathrm{a}$ is greater than that in Table 2b, and degree of rise of son's status in 1955 is higher than that in 1975, (ii) if that is included within the second quadrant, we can see that the degree of departure from MH in Table $2 \mathrm{a}$ is greater than that in Table 2b, and degree of rise of son's status in 1975 is higher than that in 1955, (iii) if that is included within the third quadrant, we can see that the degree of departure from $\mathrm{MH}$ in Table $2 \mathrm{~b}$ is greater than that in Table 2a, and degree of rise of son's status in 1975 is higher than that in 1955, and (iv) if that is included within the fourth quadrant, we can see that the degree of departure from MH in Table 2b is greater than that in Table 2a, and degree of rise of son's status in 1955 is higher than that in 1975. The confidence region for $\boldsymbol{\Lambda}_{2 a}-\boldsymbol{\Lambda}_{2 b}$ is included within the third quadrant (see Figure 2 ). Thus, we see that the degree of departure from $\mathrm{MH}$ in Table $2 \mathrm{~b}$ is greater than that in Table $2 \mathrm{a}$, and degree of rise of son's status in 1975 is higher than that in 1955.

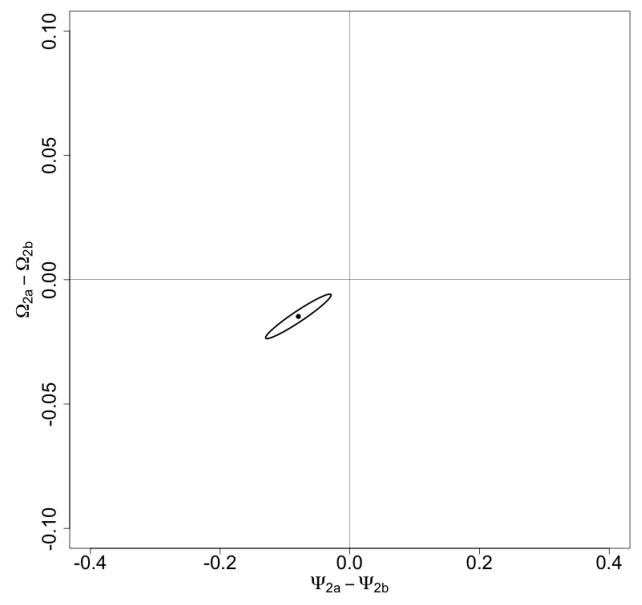

Figure 2. Approximate $95 \%$ confidence region for $\boldsymbol{\Lambda}_{2 a}-\boldsymbol{\Lambda}_{2 b}$.

\subsection{Example 2}

Consider the artificial data in Tables $3 \mathrm{a}, 3 \mathrm{~b}$ and $3 \mathrm{c}$ to show that the two-dimensional measure vector is useful for comparing visually the degree of departure from MH. From Tables $4 \mathrm{a}, 4 \mathrm{~b}$ and $4 \mathrm{c}$, we see that all of the value $\widehat{\Omega}$ for Tables $3 \mathrm{a}, 3 \mathrm{~b}$ and $3 \mathrm{c}$ are equal, although the data in Tables $3 \mathrm{a}, 3 \mathrm{~b}$ and $3 \mathrm{c}$ have a different structure of marginal inhomogeneity. Also, the value $\widehat{\Psi}$ for Table 3 c nearly equals to zero, although the data in Table $3 \mathrm{c}$ do not have a structure of marginal homogeneity. From Figure 3 , we see the degree of departure from $\mathrm{MH}$, while distinguishing two types of maximum marginal inhomogeneities. Thus, we see that (i) the data in Table 3a have a structure of lower-marginal inhomogeneity, (ii) the data in Table 3b have a structure of right-marginal inhomogeneity, and (iii) the data in Table 3c would have neither the structure of lower-marginal inhomogeneity nor that of right-marginal inhomogeneity. 
Table 3. Artificial data ( $n$ is sample size).

\begin{tabular}{|c|c|c|c|c|c|c|c|c|c|c|c|}
\hline \multicolumn{4}{|c|}{ (a) $n=740$} & \multicolumn{4}{|c|}{ (b) $n=740$} & \multicolumn{4}{|c|}{ (c) $n=740$} \\
\hline 10 & 10 & 10 & 10 & 10 & 300 & 10 & 10 & 10 & 10 & 10 & 10 \\
\hline 300 & 10 & 10 & 10 & 10 & 10 & 10 & 10 & 300 & 10 & 10 & 10 \\
\hline 10 & 10 & 10 & 10 & 10 & 10 & 10 & 300 & 10 & 10 & 10 & 300 \\
\hline 10 & 10 & 300 & 10 & 10 & 10 & 10 & 10 & 10 & 10 & 10 & 10 \\
\hline
\end{tabular}

Table 4. Estimates of $\Omega$ and $\Psi$, approximate standard errors for $\widehat{\Omega}$ and $\widehat{\Psi}$, and approximate $95 \%$ confidence intervals for $\Omega$ and $\Psi$, applied to Tables $3 \mathrm{a}, 3 \mathrm{~b}$ and $3 \mathrm{c}$.

\begin{tabular}{cccc}
\hline & $\begin{array}{c}\text { Estimated } \\
\text { measure }\end{array}$ & $\begin{array}{c}\text { Standard } \\
\text { error }\end{array}$ & $\begin{array}{c}\text { Confidence } \\
\text { interval }\end{array}$ \\
\hline (a) For Table 3a & & \\
$\Omega$ & 0.1092 & 0.0083 & $(0.0929,0.1255)$ \\
$\Psi$ & 0.3038 & 0.0136 & $(0.2771,0.3304)$ \\
(b) For Table 3b & & \\
$\Omega$ & 0.1092 & 0.0083 & $(0.0929,0.1255)$ \\
$\Psi$ & -0.3038 & 0.0136 & $(-0.3304,-0.2771)$ \\
(c) For Table 3c & & \\
$\Omega$ & 0.1092 & 0.0077 & $(0.0941,0.1243)$ \\
$\Psi$ & -0.0216 & 0.0160 & $(-0.0529,0.0097)$ \\
\hline
\end{tabular}

\section{Discussions}

The proposed measure vector $\boldsymbol{\Lambda}$ depends on the information about category ordering since $\boldsymbol{\Lambda}$ is based on the cumulative marginal probabilities. Now, we shall consider to reverse order of categories. by

Let $S_{i}^{X}$ and $S_{i}^{Y}$ denote the reverse cumulative marginal probabilities of $X$ and $Y$, respectively, defined

$$
S_{i}^{X}=\operatorname{Pr}(X \geq i+1)=\sum_{k=i+1}^{r} p_{k}, \quad S_{i}^{Y}=\operatorname{Pr}(Y \geq i+1)=\sum_{k=i+1}^{r} p_{\cdot k} \quad(i=1, \ldots, r-1) .
$$

These are the cumulative marginal probabilities which are taken in reverse order of categories; thus

$$
S_{i}^{X}=1-F_{i}^{X}, \quad S_{i}^{Y}=1-F_{i}^{Y} \quad(i=1, \ldots, r-1) .
$$

Using the reverse cumulative marginal probabilities $\left\{S_{i}^{X}, S_{i}^{Y}\right\}$, the MH model may further be expressed as

$$
S_{i}^{X}=S_{i}^{Y} \quad(i=1, \ldots, r-1)
$$

Let

$$
S_{1(i)}^{*}=\frac{S_{i}^{X}}{\widetilde{\Delta}}, S_{2(i)}^{*}=\frac{S_{i}^{Y}}{\widetilde{\Delta}}, \widetilde{Q}_{i}^{*}=\frac{1}{2}\left(S_{1(i)}^{*}+S_{2(i)}^{*}\right) \quad(i=1, \ldots, r-1)
$$

where

$$
\widetilde{\Delta}=\sum_{i=1}^{r-1}\left(S_{i}^{X}+S_{i}^{Y}\right)
$$

Tahata et al. (2006) proposed a measure based on the reverse cumulative marginal probabilities (denoted by $\widetilde{\Omega}$ ) which represents the degree of departure from MH besides the measure $\Omega$. The $\widetilde{\Omega}$ is defined by $\Omega$ with $\left\{F_{1(i)}^{*}\right\},\left\{F_{2(i)}^{*}\right\}$, and $\left\{Q_{i}^{*}\right\}$ replaced by $\left\{S_{1(i)}^{*}\right\},\left\{S_{2(i)}^{*}\right\}$, and $\left\{\widetilde{Q}_{i}^{*}\right\}$, respectively. Thus, the characteristics of $\widetilde{\Omega}$ is similar to $\Omega$. However, the definition about the maximum of the degree of departure from $\mathrm{MH}$ is $S_{i}^{X}=0$ (then $S_{i}^{Y} \neq 0$ ) for all $i=1, \cdots, r-1$ (say, maximum upper-marginal inhomogeneity) 


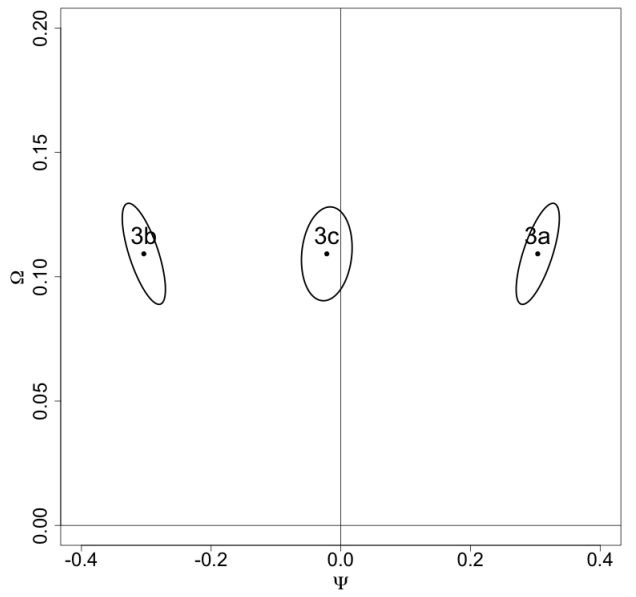

Figure 3. Approximate $95 \%$ confidence regions for $\boldsymbol{\Lambda}$, applied to Tables 3a, 3b and 3c.

or $S_{i}^{Y}=0$ (then $S_{i}^{X} \neq 0$ ) for all $i=1, \cdots, r-1$ (say, maximum left-marginal inhomogeneity). For example, the measure $\widetilde{\Omega}$ takes the maximum value 1 in the sense of maximum upper-marginal inhomogeneity and maximum left-marginal inhomogeneity for the cases in Tables 1c and 1d, respectively.

Tahata et al. (2012) proposed a measure based on the reverse cumulative marginal probabilities (denoted by $\widetilde{\Psi}$ ) which can distinguish above two types of maximum marginal inhomogeneities (namely, maximum upper-marginal inhomogeneity or maximum left-marginal inhomogeneity) besides the measure $\Psi$. The $\widetilde{\Psi}$ is defined by $\Psi$ with $\left\{F_{1(i)}^{*}\right\}$ and $\left\{F_{2(i)}^{*}\right\}$ replaced by $\left\{S_{1(i)}^{*}\right\}$ and $\left\{S_{2(i)}^{*}\right\}$, respectively. Therefore, two-dimensional measure vector $\widetilde{\boldsymbol{\Lambda}}$ with above measures $\widetilde{\Omega}$ and $\widetilde{\Psi}$ as the elements can simultaneously analyze the degree of departure from $\mathrm{MH}$ and directionality for alternative two types of maximum marginal inhomogeneities (i.e., maximum upper-marginal inhomogeneity or maximum left-marginal inhomogeneity).

Also, because of the definition of the reverse cumulative marginal probabilities, we see that the measure vector $\boldsymbol{\Lambda}$ can measure the directionality for alternative two types of maximum marginal inhomogeneities (i.e., maximum upper-marginal inhomogeneity or maximum left-marginal inhomogeneity) if we apply reverse order for categories in the table.

\section{Concluding Remarks}

For the analysis of square contingency tables with ordered categories, the proposed measure vector can represent the degree of departure from the MH model including its directionality for two types of maximum marginal inhomogeneities (i.e., maximum lower-marginal inhomogeneity or maximum rightmarginal inhomogeneity). And, it is useful for comparing visually the degree of departure from $\mathrm{MH}$ in several tables.

Acknowledgments. The authors would like to thank the referee for their helpful comments.

\section{References}

1. Agresti, A (2013). Categorical Data Analysis, 3rd Edition. Hoboken, NJ: Wiley.

2. Ando, S., Tahata, K., and Tomizawa, S. (2017). Visualized measure vector of departure from symmetry for square contingency tables. Statistics in Biopharmaceutical Research, 9, 212-224.

3. Bowker, A. H. (1948). A test for symmetry in contingency tables. Journal of the American Statistical Association. 43, 572-574. 
4. Stuart, A. (1955). A test for homogeneity of the marginal distributions in a two-way classification. Biometrika, 42, 412-416.

5. Tahata, K., Iwashita, T., and Tomizawa, S. (2006). Measure of departure from symmetry of cumulative marginal probabilities for square contingency tables with ordered categories. SUT Journal of Mathematics, 42, 7-29.

6. Tahata, K., Kawasaki, K., and Tomizawa, S. (2012). Asymmetry index on marginal homogeneity for square contingency tables with ordered categories. Open Journal of Statistics, 2, 198-203.

7. Tahata, K., Miyazawa, K., and Tomizawa, S. (2010). Measure of departure from average cumulative symmetry for square contingency tables. American Journal of Biostatistics, 1, 62-66.

8. Tominaga, K. (1979). Nippon no Kaisou Kouzou (Japanese Hierarchical Structure). University of Tokyo Press, Tokyo, (in Japanese).

9. Tomizawa, S., Miyamoto, N., and Hatanaka, Y. (2001). Measure of asymmetry for square contingency tables having ordered categories. Australian and New Zealand Journal of Statistics, 43, 335-349. 